\title{
The hidden risk: Incorporating inflammation and HIV serostatus into coronary artery disease screening
}

\author{
Gregory A. Payne, MD, PhD, a and Edgar Turner Overton, MD $^{\mathrm{b}}$ \\ a Division of Cardiovascular Disease, University of Alabama at Birmingham, School of Medicine, \\ Birmingham \\ b Division of Infectious Diseases, University of Alabama at Birmingham, Birmingham, AL
}

Received Nov 2, 2016; accepted Nov 3, 2016

doi:10.1007/s12350-016-0731-9

CAD is a well-established comorbidity associated with HIV infection. This association is in large part due to ongoing inflammation propagated by viremia and dysregulation of the immune system. Despite this knowledge, evidence to guide clinical management and screening for CAD among HIV-infected patients is lacking. The following editorial discusses recent evidence that HIV-infected patients with abnormal cardiovascular stress testing are more likely to undergo subsequent percutaneous coronary intervention. Importantly, the cardiovascular consequences of HIV infection and potential clinical implications are discussed.

Key Words: Human immunodeficiency virus $\cdot$ coronary artery disease $\cdot$ pharmacologic stress $\cdot$ exercise: stress testing $\cdot$ inflammation

\section{See related article, pp. 872-883}

As a result of robust basic and translational science, vascular inflammation is accepted as a hallmark of cardiovascular diseases including coronary artery disease (CAD). ${ }^{1}$ Despite these achievements, integration of this knowledge to clinical practice continues to lag behind. Aside from clinical associations between C-reactive protein (CRP) concentration and $\mathrm{CAD},{ }^{2,3}$ few therapeutic targets or consequential biomarkers have been discovered. Perhaps even more disappointing is the scarcity of evidence to guide clinical management of patients with known inflammatory conditions. This delayed clinical translation has never been more evident

Reprint requests: Gregory A. Payne, MD, PhD, Division of Cardiovascular Disease, University of Alabama at Birmingham, School of Medicine, Tinsley Harrison Tower, 1900 University Boulevard, Suite 311, Birmingham, AL 35233; gpayne@uabmc.edu J Nucl Cardiol 2018;25:884-6.

1071-3581/ $\$ 34.00$

Copyright (c) 2016 American Society of Nuclear Cardiology. than in populations of human immunodeficiency virus (HIV)-infected patients at risk for CAD.

With the introduction of antiretroviral therapies (ART), HIV infection has been transformed from a uniformly fatal disease to a manageable chronic illness, like type 2 diabetes. ${ }^{4}$ This dynamic shift in disease epidemiology has led to a growing older population of persons living with HIV and has increased awareness of disease comorbidities not historically associated with HIV infection. Of these comorbidities, the management of CAD has been a point of emphasis. Infection with HIV leads to dysregulation of the immune system resulting in immune hyperactivation ${ }^{5,6}$ and a systemic "cytokine storm.",7 Furthermore, coronary atherosclerotic lesions in HIV-infected patients are often unstable and more prone to rupture, resulting in a 1.5twofold higher incidence of acute coronary syndrome and myocardial infarction. ${ }^{8-11}$ Expectedly, these pathologic changes leave HIV-infected patients at risk for vascular injury, accelerated atherosclerosis, ${ }^{12}$ and ultimately disproportionate cardiovascular mortality. ${ }^{13}$ Finally, despite widespread use of ART, we are just now appreciating the burden of inflammation and resulting cardiovascular damage which threatens patient survival. $^{14}$ 
In light of these issues, Feinstein and colleagues provide new evidence further highlighting differences in CAD management based on HIV status. Focusing entirely within a high-risk patient cohort, the investigators hypothesized HIV infection was associated with increased CAD severity and subsequent ischemia. Specifically, the authors examined differences in HIVinfected and -uninfected individuals who had prior abnormal cardiovascular stress tests and underwent subsequent coronary angiography. While CAD severity assessed by visual estimates of coronary angiograms only showed non-significant trends, HIV infection was associated with an almost twofold greater likelihood for percutaneous coronary intervention (PCI). This observation persisted after adjustments for traditional CAD risk factors, with the notable exception of smoking data which were not reported.

Given the heightened inflammatory state associated with HIV infection, an association between HIV serostatus and rates of PCI seems intuitive. However, these observational results must be interpreted with caution. Some covariates are lacking from the data including hepatitis c virus status, tobacco, and substance use. The lack of reliable smoking history and pre-test clinical symptom burden limits the ability to fully adjust for both established CAD risk factors and clinical indications for PCI. Notably, HIV-infected patients are 2-3 times more likely to smoke ${ }^{15}$ compared to uninfected individuals. Furthermore, HIV-infected patients that smoke are disproportionately burdened with complications as the population-attributable risk of death from smoking is doubled compared to seronegative groups. ${ }^{16}$

Variances in ART and ongoing viremia further complicate these clinical observations. Specifically, initiation of ART and reduction in circulating viremia has been associated with improved endothelial function and reduced risk for CAD. ${ }^{17}$ Among HIV-infected participants of the current study, 27\% were not currently taking ART and presumptively had ongoing viral replication, an important risk factor for accelerated atherosclerosis. ${ }^{18}$ These differences introduce some uncertainty regarding the homogeneity within the HIVinfected cohort that could have influenced the observed trends toward PCI.

Despite these limitations, the investigators have potentially uncovered a "hidden" ischemic burden associated with HIV infection. The almost twofold increase in rates of PCI suggests HIV-infected participants may have had larger territories of ischemia or higher risk coronary anatomy. While all participants had abnormal (non-equivocal) cardiovascular stress tests, an interesting correlation would have been to assess the ischemic burden between HIV-infected and -uninfected individuals. Nonetheless, taking efforts to quantify disease burden within a high-risk group of HIV-infected patients is a novel approach to better understand the operative characteristics of stress tests (i.e., positive predictive values) and clinical pathology of HIV-associated CAD. As discussed in the study, the addition of more advanced invasive measures of coronary physiology (i.e., fractional flow reserve, coronary flow reserve, or index microvascular resistance) and atherosclerotic severity (i.e., intravascular ultrasound and optical coherence tomography) may further illuminate differences in CAD burden.

While these findings may underscore the need for screening of symptomatic HIV-infected individuals, we must consider how non-invasive CAD screening performs in asymptomatic HIV-infected populations. In a cohort study of African American adults who underwent coronary CT angiography (CCTA), Lai et al. recently published findings suggesting subclinical CAD was more common in HIV-infected patients on long-term ART. ${ }^{19}$ Furthermore, previous investigations also employing CCTA demonstrated an age-associated high rate of coronary stenosis ( $>50 \%$ luminal area) in asymptomatic HIV-infected subjects. ${ }^{20,21}$ Interestingly, these patients often had low cardiovascular risk (Framingham Risk Score $\leq 10$ ) and negative echocardiographic and ECG stress testing prior to anatomic evaluation with CCTA. Hence, in an era of symptom-driven ischemic evaluations, HIV-infected patients may be at high risk regardless of presenting symptom burden or functional non-invasive screening results. Therefore, continued investigations with other imaging modalities as well as optimizing cardiovascular risk calculators are needed to understand the optimal approach for CAD screening within HIV-infected populations. More importantly, there is a critical need for prospective research to evaluate cardiovascular events and clinical outcomes among patients with imaging abnormalities.

The more difficult decision confronting both cardiologists and infectious disease specialists is how to manage HIV-infected patients with abnormal cardiovascular stress tests. While the decision to proceed with PCI during acute coronary syndrome may be straightforward, can physicians safely extrapolate results from the COURAGE ${ }^{22,23}$ or ongoing ISCHEMIA Trials to HIV-infected patients with stable CAD? Additionally, the perceived benefits of optimal medical therapy may not fully extend to HIV-infected patients, which may account for continued health outcome disparities. Results from Feinstein et al. suggest that indeed significant differences may exist within HIV-infected communities, and that a "one-size-fits-all" approach to non-invasive stress testing may be inappropriate. While efforts to address these issues are ongoing, including the 
Randomized Trial to Prevent Vascular Events in HIV (REPRIEVE) Trial (NCT02344290), future studies are needed to determine the best practice for screening HIVinfected patients.

Increased utilization of non-invasive multimodality imaging technologies may be a novel approach to help correct these disparities. In particular, the ability to simultaneously assess coronary anatomy and physiology may add incremental value to risk assessments for HIVinfected patients. The combination of Rubidium-82 positron emission tomography (PET) and coronary computed tomography (CT) has recently been demonstrated to detect differences in myocardial blood flow between HIV-infected and -uninfected patients, ${ }^{24}$ suggesting the potential to identify patients with underlying endothelial dysfunction and risk for disease progression. In addition, with continued refinement of molecular imaging, the day may come when we can visualize coronary inflammation and identify HIV-infected patients at highest risk for complications. ${ }^{25}$

Work from Feinstein and colleagues highlights the need to refine CAD risk assessments and non-invasive screening of HIV-infected patients. More importantly, these findings validate our continued focus on HIV-associated complications and other "hidden" inflammatory risk factors for CAD. While new technologies may remain limited, future multimodality approaches within HIV-infected populations may help mitigate continued disparities in cardiovascular disease outcomes.

\section{Disclosure}

The authors have no financial relationships to disclose.

\section{References}

1. Libby P. Inflammation in atherosclerosis. Nature 2002;420:86874.

2. Sabatine MS, et al. Prognostic significance of the Centers for Disease Control/American Heart Association high-sensitivity Creactive protein cut points for cardiovascular and other outcomes in patients with stable coronary artery disease. Circulation 2007;115:1528-36.

3. Zebrack JS, Muhlestein JB, Horne BD, Anderson JL, Intermountain Heart Collaboration Study Group. C-reactive protein and angiographic coronary artery disease: Independent and additive predictors of risk in subjects with angina. J Am Coll Cardiol 2002;39:632-7.

4. Lesko CR, et al. The effect of antiretroviral therapy on all-cause mortality, generalized to persons diagnosed with HIV in the USA, 2009-11. Int J Epidemiol 2016;45:140-50.

5. Hunt PW, et al. T cell activation is associated with lower CD4+ T cell gains in human immunodeficiency virus-infected patients with sustained viral suppression during antiretroviral therapy. J Infect Dis 2003;187:1534-43.

6. Funderburg NT, et al. Shared monocyte subset phenotypes in HIV1 infection and in uninfected subjects with acute coronary syndrome. Blood 2003;120:4599-608.

7. Ford ES, Puronen CE, Sereti I. Immunopathogenesis of asymptomatic chronic HIV infection: The calm before the storm. Curr Opin HIV AIDS 2009;4:206-14.

8. Freiberg MS, et al. HIV infection and the risk of acute myocardial infarction. JAMA Intern Med 2013;173:614-22.

9. Hanna DB, et al. Trends in cardiovascular disease mortality among persons with HIV in New York City, 2001-2012. Clin Infect Dis 2016;63:1122-9.

10. Silverberg MJ, et al. Immunodeficiency and risk of myocardial infarction among HIV-positive individuals with access to care. J Acquir Immune Defic Syndr 2014;65:160-6.

11. Althoff $\mathrm{KN}$, et al. Comparison of risk and age at diagnosis of myocardial infarction, end-stage renal disease, and non-AIDSdefining cancer in HIV-infected versus uninfected adults. Clin Infect Dis 2015;60:627-38.

12. Hsue PY, et al. Carotid intima-media thickness progression in HIV-infected adults occurs preferentially at the carotid bifurcation and is predicted by inflammation. J Am Heart Assoc 2012;1:jah3e000422.

13. Palella FJ Jr, et al. Mortality in the highly active antiretroviral therapy era: Changing causes of death and disease in the HIV outpatient study. J Acquir Immune Defic Syndr 2006;43:27-34.

14. Lohse N, et al. Survival of persons with and without HIV infection in Denmark, 1995-2005. Ann Intern Med 2007;146:87-95.

15. Tesoriero JM, Gieryic SM, Carrascal A, Lavigne HE. Smoking among HIV positive New Yorkers: Prevalence, frequency, and opportunities for cessation. AIDS Behav 2010;14:824-35.

16. Helleberg M, et al. Mortality attributable to smoking among HIV1-infected individuals: A nationwide, population-based cohort study. Clin Infect Dis 2013;56:727-34.

17. Torriani FJ, et al. Endothelial function in human immunodeficiency virus-infected antiretroviral-naive subjects before and after starting potent antiretroviral therapy: The ACTG (AIDS Clinical Trials Group) Study 5152s. J Am Coll Cardiol 2008;52:569-76.

18. Hatano $\mathrm{H}$, et al. Prospective antiretroviral treatment of asymptomatic, HIV-1 infected controllers. PLoS Pathog 2013;9: e1003691.

19. Lai $\mathrm{H}$, et al. HIV infection itself may not be associated with subclinical coronary artery disease among African Americans without cardiovascular symptoms. J Am Heart Assoc 2016;5:e002529.

20. d'Ettorre G, et al. Significant coronary stenosis detected by coronary computed angiography in asymptomatic HIV infected subjects. J Infect 2012;64:82-8.

21. Lo $J$, et al. Increased prevalence of subclinical coronary atherosclerosis detected by coronary computed tomography angiography in HIV-infected men. AIDS 2010;24:243-53.

22. Boden WE, et al. Optimal medical therapy with or without PCI for stable coronary disease. N Engl J Med 2007;356:1503-16.

23. Sedlis SP, et al. Effect of PCI on long-term survival in patients with stable ischemic heart disease. N Engl J Med 2015;373:193746.

24. Knudsen A, et al. Normal myocardial flow reserve in HIV-infected patients on stable antiretroviral therapy: A cross-sectional study using Rubidium-82 PET/CT. Medicine 2015;94:e1886.

25. Jaffer FA, Libby $P$, Weissleder R. Molecular imaging of cardiovascular disease. Circulation 2007;116:1052-61. 